\title{
Adhesive aero-hydrogel hybrid conductor assembled from silver nanowire architectures
}

\author{
Zhao Pan ${ }^{1}, \mathrm{Ze}-\mathrm{Yu}$ Wang ${ }^{1}$, Mo-Han Wang ${ }^{2}$, Lu Yang ${ }^{2}$ and Shu-Hong Yu ${ }^{1 *}$
}

\begin{abstract}
Conductive and adhesive hydrogels are promising materials for designing bioelectronics. To satisfy the high conductivity of bioelectronic devices, metal nanomaterials have been used to fabricate composite hydrogels. However, the fabrication of a conductive-nanomaterial-incorporated hydrogel with high performance is a great challenge because of the easy aggregation nature of conductive nanomaterials making processing difficult. Here, we report a kind of adhesive aero-hydrogel hybrid conductor (AAHC) with stretchable, adhesive and anti-bacteria properties by in situ formation of a hydrogel network in the aerogel-silver nanowires (AgNWs) assembly. The AgNWs with good conductivity are wellintegrated on the inner-surface of shape-memory chitosan aerogel, which created a conductive framework to allow hydrogel back-filling. Reinforcement by the aerogel-silver makes the hybrid hydrogel tough and stretchable. Functional groups from the hydrogel allow strong adhesion to wet tissues through molecular stitches. The inherent bacteria-killing ability of silver ions endows the conductive hydrogel with excellent anti-bacteria performance. The proposed facile strategy of aerogel-assisted assembly of metal nanomaterials with hydrogel opens a new route to incorporate functional nanoscale building blocks into hydrogels.
\end{abstract}

Keywords: silver nanowire, chitosan aerogel, templating assembly, conductive hydrogel

\section{INTRODUCTION}

Wearable and implantable bioelectronic devices, which are designed to interface with human body, have been intensely studied to collect and deliver various bioelectronic signals on different human body parts such as brain, spinal cord, skin and heart [1-5]. Despite the huge promise in personalized healthcare that bioelectronics can offer, the stark disparities between rigid conventional electrodes and soft, high water-containing tissues, pose immense challenges towards clinical operations and postoperation care $[3,6]$.

In light of their similar mechanical properties toward biological tissues in human body, hydrogels have been receiving a great deal of attention for applications as bioelectronics [6-9]. Despite their high compliance, toughness, and stretchability, conductive properties of hydrogel are typically provided by requisite additives $[10,11]$. An efficient method to endow hydrogels with high conductivity involves integration of conventional conductors including conducting polymers [11], carbonbased nanomaterials [12,13], and nanometallic materials into the hydrogel network [14]. As a promising candidate additive, silver nanowires (AgNWs) with excellent conductivity and anti-bacterial performances have drawn great attention for building elastic conductors [15-21]. However, while harnessing unique advantages of AgNWs, either the random distribution or easy aggregation of the nanomaterials in a polymer matrix leads to poor conductivity and weak mechanical strength, which causes the shrinkage of their application [22,23]. Hence, it is highly desirable, from the viewpoint of practical applications, to develop an effective assembling strategy for integration of one-dimensional conductive nanostructures into a macroscopic flexible hydrogel conductor.

Engineering bio-devices typically requires robust fixation on tissues for their corresponding functional purpose [24]. To avoid physical damage induced by mechanical fixation such as suturing [25], and prevent delamination failure of the bio-electronic device from tissues, the interfacial adhesion between bioelectronics and tissues is of critical importance. Thus, an effective adhesive mechan-

\footnotetext{
${ }^{1}$ Division of Nanomaterials \& Chemistry, Hefei National Laboratory for Physical Sciences at the Microscale, Institute of Energy, Hefei Comprehensive National Science Center, CAS Center for Excellence in Nanoscience, Department of Chemistry, Institute of Biomimetic Materials \& Chemistry, Anhui Engineering Laboratory of Biomimetic Materials, University of Science and Technology of China, Hefei 230026, China

${ }^{2}$ Stomatology Hospital \& College, Anhui Medical University, Key Laboratory of Oral Diseases Research of Anhui Province, Hefei 230032, China * Corresponding author (email: shyu@ustc.edu.cn)
} 
ism is strongly required in designing hydrogel electronics. Recently, tough bonding of hydrogels on diverse wet tissues has been proposed by topologically anchoring hydrogel through molecular stitches [26]. Such robust interfacial adhesion can offer more reliable bio-electronic performances and functionalities, particularly for dynamic and highly deformable tissues such as skin.

Herein, we report the fabrication of a flexible hydrogel conductor based on a chitosan (CTS) aerogel-assisted AgNW assembly and in situ hydrogel polymerization strategy. The adhesive aero-hydrogel hybrid conductor (AAHC) is firstly fabricated from the ice-templated CTS aerogel that offers a rigid framework to integrate AgNWs into the conductive network and provides mechanical supporting for AAHC. In addition, the backfilling poly (acrylamide-sodium acrylate) (PAMSA) hydrogel works as soft matrix to tack AAHC on tissues and dissipate energy when AAHC is under stress. Consequently, AAHC bioelectronics features high electronic conductivity, superior mechanical properties, anti-bacteria capability and excellent tissue adhesive performance.

\section{EXPERIMENTAL SECTION}

\section{Preparation of porous CTS aerogel}

CTS solution ( $2 \mathrm{wt} \%$ ) was prepared by dissolving CTS powder $(1.0 \mathrm{~g})$ in $50 \mathrm{~mL}$ of acetic acid aqueous solution (2 wt\%). CTS scaffolds were fabricated according to the previously reported ice-templated process [27]. Briefly, viscous polymer solution was frozen in a silicon rubber mold placed on the top of pre-cooled steel plate. The obtained frozen samples were then freeze-dried by using a Labconco-195 freeze-drier.

\section{Preparation of AgNW attached CTS aerogel (Ag-CTS aerogel)}

AgNWs were synthesized in a modified process reported previously [28]. In brief, $5.86 \mathrm{~g}$ of poly(vinylpyrrolidone) (PVP K30) and $190 \mathrm{~mL}$ of glycerol were added into a $500-\mathrm{mL}$ round bottom flask and dissolved at $100^{\circ} \mathrm{C}$ to form a homogenous solution. After being cooled to room temperature, $1.58 \mathrm{~g}$ of $\mathrm{AgNO}_{3}$ powder was added into the flask. With gentle stirring, the solution was heated to $210^{\circ} \mathrm{C}$ for $30 \mathrm{~min}$. During the heating process, $10 \mathrm{~mL}$ of glycerol solution containing $59 \mathrm{mg}$ of $\mathrm{NaCl}$ and $0.5 \mathrm{~mL}$ of $\mathrm{H}_{2} \mathrm{O}$ was added at $60^{\circ} \mathrm{C}$. The reaction was immediately stopped after the temperature reached $210^{\circ} \mathrm{C}$ by adding $200 \mathrm{~mL}$ of room-temperature deionized water. The color of the reaction solution evolved from pale white to light brown, red, dark gray, and eventually grayish-green. The resultant AgNWs were washed with deionized water twice before use. Then, the freeze-dried CTS aerogel was immersed in aqueous ammonia to neutralize the acid and rinsed with water three times. After that, the CTS aerogel was immersed in AgNW dispersion, squeezed and then released. After repeating squeezing-releasing cycles five times, the resultant Ag-CTS aerogel was freeze-dried in a Labconco-195 freeze-drier.

\section{Preparation of adhesive AAHC}

Firstly, $7.2 \mathrm{~g}$ acrylic acid was neutralized by $25 \mathrm{~mL}$ of $4 \mathrm{~mol} \mathrm{~L}^{-1} \mathrm{NaOH}$ aqueous solution and cooled down to room temperature. Then, $3.55 \mathrm{~g}$ acrylamide was added to make stock A solution. The pre-gel solution was made by mixing $3.5 \mathrm{~mL}$ stock $\mathrm{A}, 2.5 \mathrm{~mL}$ deionized water, $110 \mu \mathrm{L}$ $N, N^{\prime}$-methylenebisacrylamide solution (2 wt\% aqueous solution), $100 \mu \mathrm{L}$ ammonium persulphate (10 wt $\%$ aqueous solution) and $10 \mu \mathrm{L} \quad N, N, N^{\prime}, N^{\prime}$-tetramethylethylenediamine. Then, Ag-CTS aerogels were immersed inside the pre-gel solution to infiltrate the aerogel with the hydrogel precursor, followed by degassing in a vacuum oven and curing at $40^{\circ} \mathrm{C}$ for $1 \mathrm{~h}$ under nitrogen atmosphere. The resultant hybrid conductor was thoroughly rinsed with a large amount of deionized water for $24 \mathrm{~h}$.

\section{In vitro cytocompatibility test}

The cytocompatibility of materials was assessed by the CCK-8 kit. In brief, the material samples were incubated in cell culture medium at $37^{\circ} \mathrm{C}$ for $24 \mathrm{~h}$ to prepare conditioned medium. Mouse fibroblasts (NIH/3T3) were plated in 96-well plates $\left(2.0 \times 10^{4}\right.$ cells per well, $N=4$ per group) and cultivated with $200 \mu \mathrm{L}$ per well normal culture medium or each conditioned medium for $24 \mathrm{~h}, 3,5$, and 7 days, respectively. Cell proliferation was determined by a CCK-8 assay. CCK-8 reagent was mixed with Dulbecco's modified Eagle medium (DMEM) at 1:10 and added into the wells $(110 \mu \mathrm{L}$ per well). Then the cells were cultivated for another $2 \mathrm{~h}$ at $37^{\circ} \mathrm{C}$. Finally, the culture medium was removed and the viability was measured by a microplate reader at a wavelength of $450 \mathrm{~nm}$.

\section{Anti-bacteria performance test}

Staphylococcus aureus (S. aureus, Gram-positive, ATCC 25923) and Escherichia coli (E. coli, Gram-negative, ATCC 25922) were employed to evaluate the antimicrobial activity of the materials. The antibacterial properties were determined by optical density value at $600 \mathrm{~nm}\left(\mathrm{OD}_{600}\right)$. All the microbial cell suspensions were diluted to $10^{5} \mathrm{CFU} \mathrm{mL}{ }^{-1}$. The materials were punched 
into uniform discs of $6 \mathrm{~mm}$ diameter and then were sterilized using a high-pressure steam sterilizer (HVE-50, HIRAYAMA company, Japan). Ten microliter of bacterial culture was taken out after $12 \mathrm{~h}$ incubation, and diluted with the corresponding medium until $\mathrm{OD}_{600}$ value came to around $0.08\left(\mathrm{OD}_{600}\right.$ of culture medium is 0.072$)$. One material disk was immersed in each culture tube and incubated at $37^{\circ} \mathrm{C}$, and $100 \mu \mathrm{L}$ of the culture medium was taken out for testing on the micro-plate reader at setting time points $(0,1,2,3,4,6,8,10$ and $12 \mathrm{~h})$. A group of culturing bacteria without the material was used as a control. The growth curve of the corresponding bacteria was depicted according to the OD value of the bacterial culture. All the experiments were carried out in triplicate.

\section{In vivo biocompatibility test}

Animal studies were performed according to the Guide for the Care and Use of Laboratory Animals of the National Institutes of Health. The in vivo biocompatibility test was in full compliance with the guidelines of the Institutional Animal Care and Use Committee of Anhui Medical University, China (LLSC20150002). Specific pathogen-free (SPF) Sprague-Dawley rats (male, 7-8 weeks) were purchased from Anhui Medical University. Animals were randomly selected for operation without blinding or applied inclusion and exclusion criteria. Rats were housed singly in plastic cages containing sterile bedding in ventilated animal rooms and maintained on a $12 / 12 \mathrm{~h}$ light/ dark cycle with sterile food and water. Prior to implantation, $10 \mathrm{~mm} \times 10 \mathrm{~mm} \times 1 \mathrm{~mm}$ rectangular samples were prepared using sterile techniques and were further sterilized for $3 \mathrm{~h}$ under ultraviolet light. The rats were anesthetized with pentobarbital, dorsal hairs were removed and the animals were placed over a heating pad for the duration of the surgery. The subcutaneous space was accessed by a 1-2 cm skin incision per implant in the center of the animals' back. To create space for implant placement, blunt dissection was performed from the incision towards the animal shoulder blades. The materials were placed in the subcutaneous pocket created above the incision, and then the incision was closed using interrupted suture. Up to two implants were placed per animal ensuring no overlap between each subcutaneous pocket created. After two weeks following the implantation, the animals were euthanized by $\mathrm{CO}_{2}$ inhalation. Subcutaneous regions of interest were excised and fixed in $10 \%$ formalin for $24 \mathrm{~h}$ for histological analyses.

\section{Characterization}

Scanning electron microscopy (SEM) images of the freeze-dried samples were carried out on a field emission scanning electron micro-analyzer (Zeiss Supra 40, Carl Zeiss AG, Baden-Wurttemberg, Oberkochen, Germany). The morphologies of the samples were observed using a Hitachi H7700 transmission electron microscope (TEM) at an acceleration voltage of $120 \mathrm{kV}$. The electrical conductivity of aerogels, hydrogels and hybrid composites was measured by a two-probe method with the PM5 Analytical Probe System (Cascade Microtech, Inc., Beaverton, OR, USA) and Keithley 4200 SCS at room temperature in air. Stretch tests with different strains were carried out on the Instron 5565A. The sample size was measured using a standard caliper. The adhesive tests were also carried out on the Instron 5565A following the instructions of ASTM F2256.

\section{RESULTS AND DISCUSSION}

\section{Preparation of robust AAHC}

Fig. 1a schematically illustrated the fabrication of a highly conductive, stretchable hybrid conductor through backfilling hydrogel into AgNW attached CTS framework. In brief, firstly, the unidirectional CTS aerogel was constructed by a ice-templated freeze casting process (Fig. 1b). As shown in the top-view SEM image in Fig. 1f, the CTS aerogel demonstrated an undisrupted cellular structure that interconnected through porous compartmental walls (Fig. 1g). After being neutralized by immersing in aqueous ammonia for $1 \mathrm{~h}$ and washed with pure water, this CTS aerogel exhibited robust mechanical properties and highly charged inner-surfaces due to the abundant amino groups on CTS molecule skeleton. The uniaxially aligned and interconnected tunnels endow the aerogel with excellent resistance towards compression, and their structure can recover fast while immersed in water (shape-memory property) [27]. These unique structure-allowed properties provide an ideal platform for fabricating flexible bioelectronics.

High aspect-ratio AgNWs were facilely synthesized through a template method. In glycerol, silver ions assembled along the axis with the constraint of PVP (polyvinylpyrrolidone, K30) to form negatively charged nanowires (Fig. S1a-c). Through simply immersing the as-prepared CTS aerogel into AgNW aqueous dispersion and repeating compressing-releasing cycles, the AgNWs could be easily fixed onto the porous CTS framework through electrostatic adsorption and integrated into conductive networks (Fig. 1c and Fig. S1d). As shown in the SEM image (Fig. 1h, i), the smooth walls of CTS aerogel (Fig. S2a) were overlaid with AgNWs after at- 


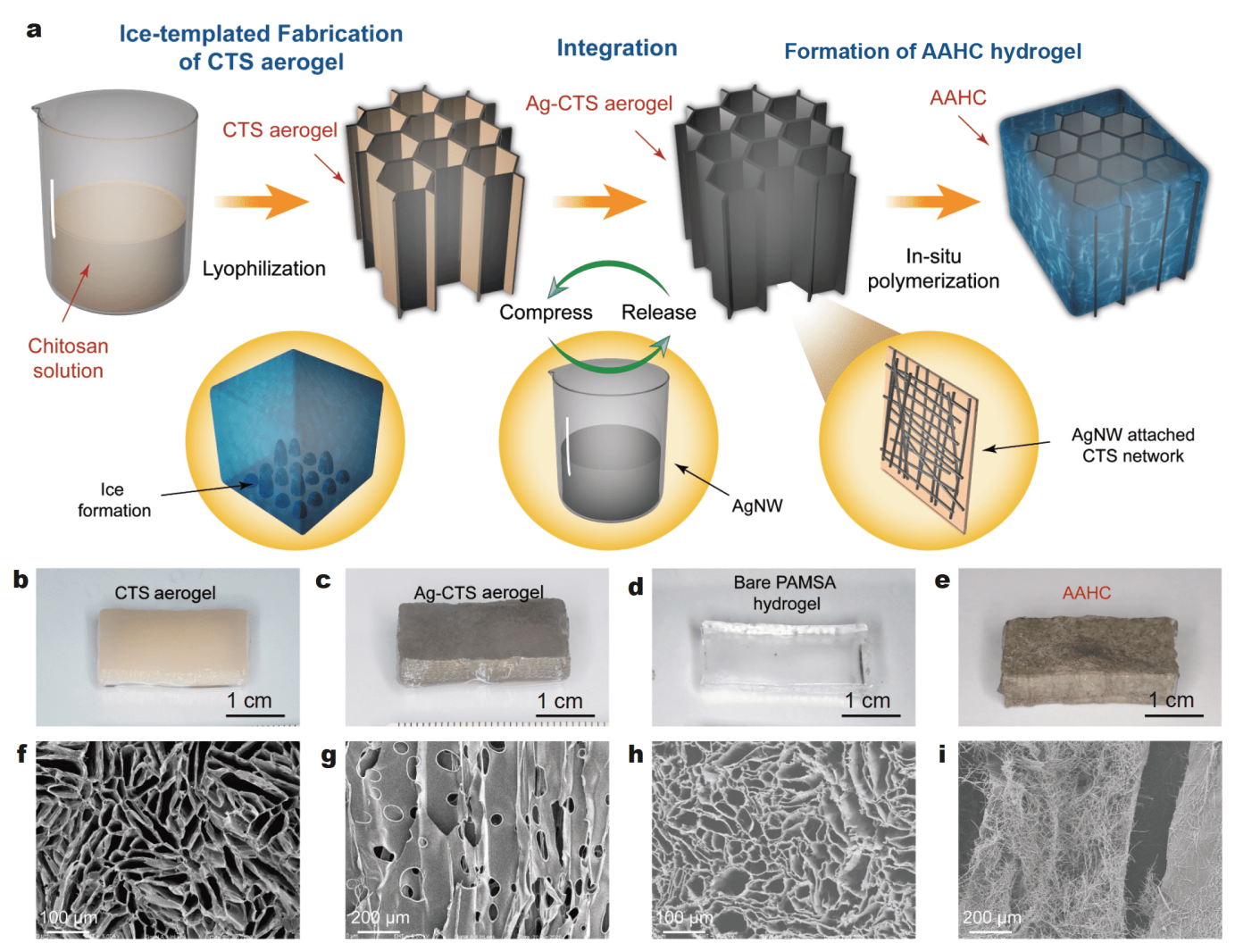

Figure 1 Fabrication procedure and morphology of the AAHC. (a) Schematic illustrations of the preparation of AAHC. Typically, CTS aerogel with a cellular structure was prefabricated by the lyophilization method. With recycling resorption, the AgNWs were integrated onto the surface of CTS and formed an interconnected network. The AAHC was finally fabricated after in-situ polymerization of PAMSA hydrogel. The digital pictures of (b) CTS aerogel, (c) Ag-CTS aerogel, (d) PAMSA hydrogel, and (e) AAHC were further demonstrated. SEM images of CTS aerogel (f, g) before and (h, i) after integrating AgNWs.

taching cycles (Fig. S2b), and meanwhile, the structural integration of CTS aerogel was perfectly maintained. Compared with conventional ice-templated methods for metal nanowires assembly [15], this two-step approach not only facilitates inter-connected AgNW network on the inner surface of aerogel, but more importantly, the natural polymer-based framework also provides great mechanical properties to facilitate establishment of robust conductors.

At the last stage to prepare AAHC, the pre-gel solution of PAMSA hydrogel was penetrated into the aerogel through vacuum infusion. Due to the strong negative charges inherited from the sodium acrylate monomer, the subsequent hydrogel network presents high affinity with the CTS framework and attached AgNWs. The interactions between building blocks of CTS, AgNWs and hydrogels integrate the bulk material into a robust composite bioelectronics. Compared with homogenous matrix formed from the pure PAMSA hydrogel (Fig. S2c), the Ag-CTS aerogel exhibits a uniform filling of hydrogel inside without disrupting its integrity, as revealed by the SEM image (Fig. S2d).

\section{Mechanical and adhesive performances}

Hydrogel network can be uniformly distributed in the aerogel framework because the monomer possesses abundant functional groups like the carboxylate group, which interact with the CTS framework by noncovalent bonding and electrostatic interactions. Incorporation of PAMSA hydrogel into the CTS aerogel combined the high stretchability of PAMSA hydrogel and strength of Ag-CTS aerogel together, endowed the composites with high stretchability, toughness and excellent recovery capability. Typical uniaxial tensile stress-strain curves showed that the tensile strength of the AAHC was $70 \mathrm{kPa}$, which is significantly higher than that of the Ag-CTS aerogel $(45 \mathrm{kPa})$ and PAMSA hydrogel (10 kPa) (Fig. 2a, $\mathrm{b})$; moreover, due to the strong bonding formed between the aerogel framework and hydrogel network, the maximum tensile strain of the hybrid (200\%) is four times 

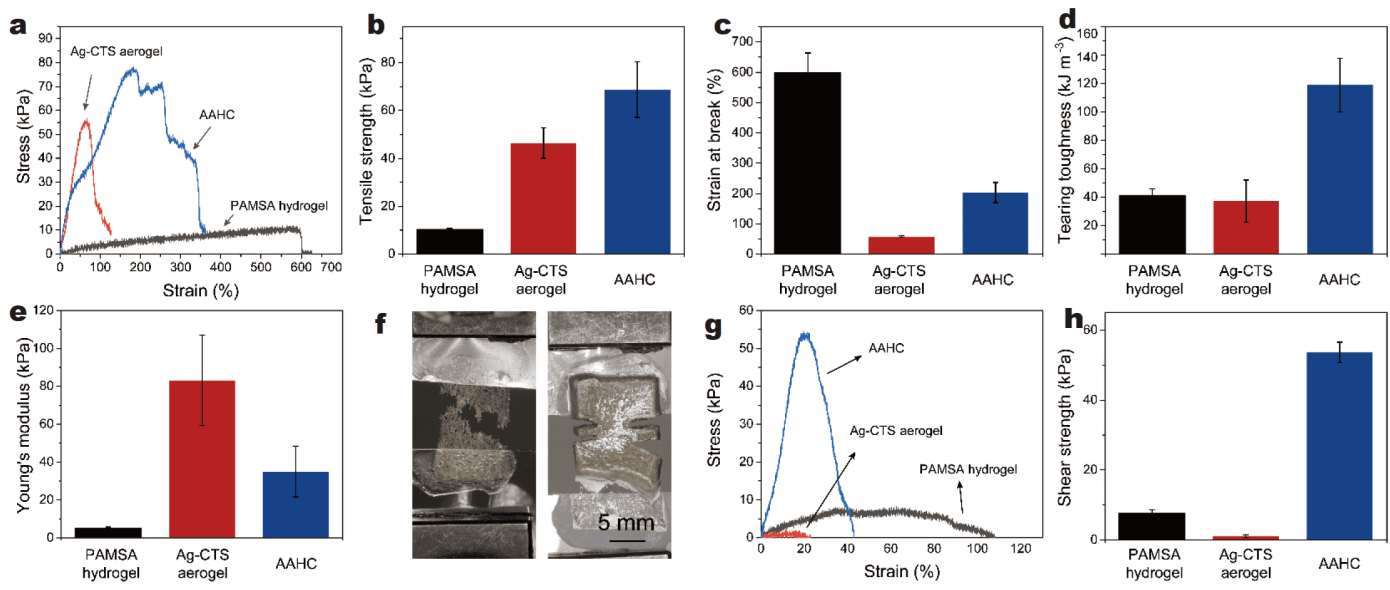

Figure 2 Mechanical and adhesive performances. (a) Typical tensile stress-strain curves, (b) tensile strength, (c) breaking strain, (d) tearing toughness and (e) Young's modulus of Ag-CTS aerogel, PAMSA hydrogel and AAHC. (f) Photographs demonstrating different ways of tearing between Ag-CTS aerogel and AAHC. (g) Typical shear-lap curves and (h) shear strength of Ag-CTS aerogel, PAMSA hydrogel and AAHC adhering on wet biological surface. Error bars represent standard deviation (SD); $N=4$.

that of pristine aerogel (50\%) (Fig. 2c). Additionally, this strong interfacial bonding together with the energydissipation capability of hydrogel matrix led to a synergistic effect in toughness of the hybrid (Fig. 2d) - the tearing toughness of AAHC is clearly enhanced when compared with that of the two components. Due to the parallel tunnel structure, for the Ag-CTS aerogel, only the fracture of ice-templated walls is responsible for the fracture resistance and energy dissipation during tearing, leading to very limited tearing toughness. In comparison, when integrated with PAMSA hydrogel, the fracture of ice-templated walls occurred after the deformation and fracture of very flexible hydrogel matrix, resulting in a composite with exceptionally high tear resistance. Besides the toughness, for the sake of interfacial and mechanical stability, wearable and implantable electronics should match the elastic modulus of surrounding biological tissues [6]. As shown in Fig. 2e, different from the squishy pure PAMSA hydrogel (Young's modulus $5 \mathrm{kPa}$ ) and stiff Ag-CTS aerogel (Young's modulus $80 \mathrm{kPa}$ ), the elastic modulus of AAHC can be easily tuned into a moderate range $(30 \pm 10 \mathrm{kPa})$. Therefore, the as-prepared aerohydrogel hybrid has shed a light on the design of electronic devices resembling natural tissues, which undoubtedly increases their compatibility with biological systems.

For either electronic skin or implantable bio-electrodes, interfacial adhesion between the device and tissues is of critical importance to avoid damage towards both electronics and dynamic tissues. In this scenario, it is desirable to develop flexible and tissue-adhesive bioelectronics so that they can tightly integrate with surrounding tissues. Recently, tissue adhesives based on topological adhesion mechanism have attracted extensive research attention [29]. Here we design an approach that molecularly stitches wet AAHCs to tissues. CTS molecular chains were used as stitches to bond to the surface of each adherends, and then form a new polymer network at the interface, resulting in strong wet adhesion between AAHC and tissues. After CTS solution was applied, the stitch molecules penetrated into and topologically entangled with the network of adherends' surface. Besides, additives in the solution, 1-ethyl-3-(3-dimethylaminopropyl) carbodiimide hydrochloride (EDC) and N-hydroxy succinimide (NHS), facilitate the stitch molecules to form chemical bonds with functional groups of adherends, like amino and carboxyl groups. Quantitative tensile-adhesion testing revealed that the hybrid conductors adhered strongly to test tissue substitution (wet porcine sausage skin membrane), and their adhesive strength $(55 \mathrm{kPa})$ is comparable to those of commercially available sealants such as Histoacryl and Coseal [30,31]. Notably, combining tough interfacial bonding and efficiently enhanced matrix energy-dissipating ability, the adhesion strength of hybrid conductors became prominently stronger than that of Ag-CTS aerogel $(3 \mathrm{kPa})$ and PAMSA hydrogel $(8 \mathrm{kPa})$.

\section{Electromechanical property}

The structural advantages of the 3D binary-network architecture and extraordinary electrical conductivity combined with the excellent mechanical robustness of 
hydrogel make the hybrid an appropriate candidate for use as flexible and stretchable bioelectronics. The in situ formation of AgNW network on CTS framework resulted in high electrical conductivity of the aerogel (Fig. 3a). The CTS aerogel and PAMSA hydrogel are inherently insulating, but the hybrid after combination with PAMSA hydrogel showed good electrical conductivity, suggesting that the formation of hydrogel surrounding AgNWs caused little damage to the conductive network. In addition, the resistance variation as a function of uniaxial tensile strain from $15 \%$ to $30 \%$ was then investigated. As revealed in Fig. 3b, c, the resistance of the aero-hydrogel hybrid increases with the increasing tensile strain during the stretching cycles, exhibiting excellent sensitivity, response capability and repeatability. Even after one-thousand stretch-release cycles (Fig. 3d), the hybrid did not show any obvious irreversible resistance increase. These results are a proper demonstration of the excellent electromechanical stability of aero-hydrogel hybrids and their great potential for high-performance stretchable and flexible bioelectronics.

\section{Biocompatible performances}

As the hybrid has a good conductive capacity and tissue adhesive performance, it is necessary to estimate the cy- tocompatibility of it for potential biomedical application. To explore the cytocompatibility of the hybrid, we used the fibroblasts for cell proliferation analysis and demonstrated the biocompatibility of CTS framework, CTSAgNW aerogel, and hybrid to fibroblasts by live/death analysis. To be sure, the in vitro biocompatibility of the hybrid-conditioned medium is comparable to that of the control medium, CTS framework medium and CTSAgNW aerogel-conditioned medium, showing no observable decrease in the in vitro viability of the blasts after 24-h, 3-d, 5-d, and 7-d culture (Fig. 4a). The fluorescence images (Fig. $4 \mathrm{~b}$ ) showed no decrease in the in vitro viability of fibroblasts after 24-h culture.

Implantation of bioelectronics could cause damage to tissues and easily recruit surrounding microbial, triggering wound infection and local inflammation. Therefore, bioelectronics with antibacterial ability is of great value during the clinical application. The antibacterial properties of our hybrid were tested by using E. coli and $S$. aureus as the representative bacteria. For comparison, CTS framework and CTS-AgNW aerogel were also committed to antibacterial test by co-culturing with bacteria. Pre-cultured E. coli and S. aureus were cultured on an automated shaker at $37^{\circ} \mathrm{C}$ containing the experimental materials, respectively. The $\mathrm{OD}_{600}$ value of the
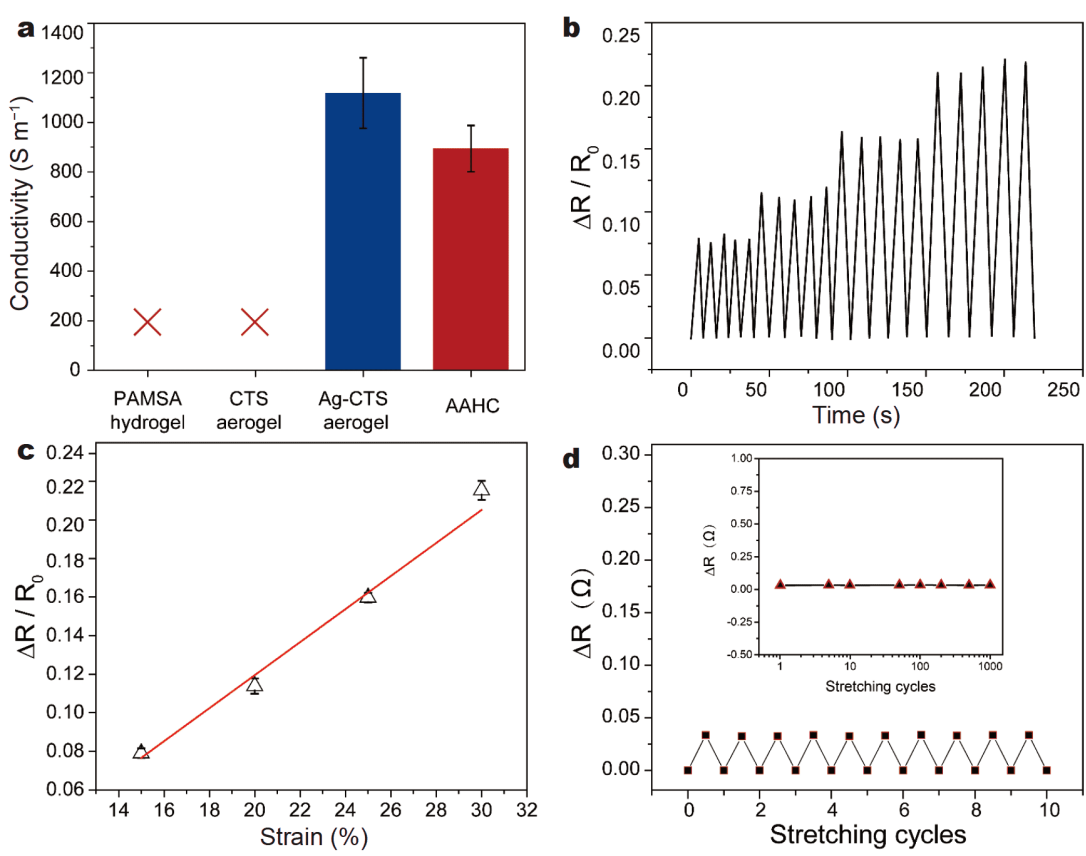

Figure 3 Electrical performance of AAHC. (a) Conductivity of PAMSA hydrogel, CTS aerogel, Ag-CTS aerogel and AAHC. (b) Sensitivity, response capability, repeatability test of AAHC at $15 \%, 20 \%, 25 \%, 30 \%$. (c) Resistance changes caused by the transverse tension as a function of true strain. (d) $\Delta R$ values of AAHC during the stretching-releasing cycles with a small distance of $2 \mathrm{~mm}$. The inset shows the $\Delta R$ values during 1000 cycles. Error bars represent $\mathrm{SD} ; \mathrm{N}=4$. 

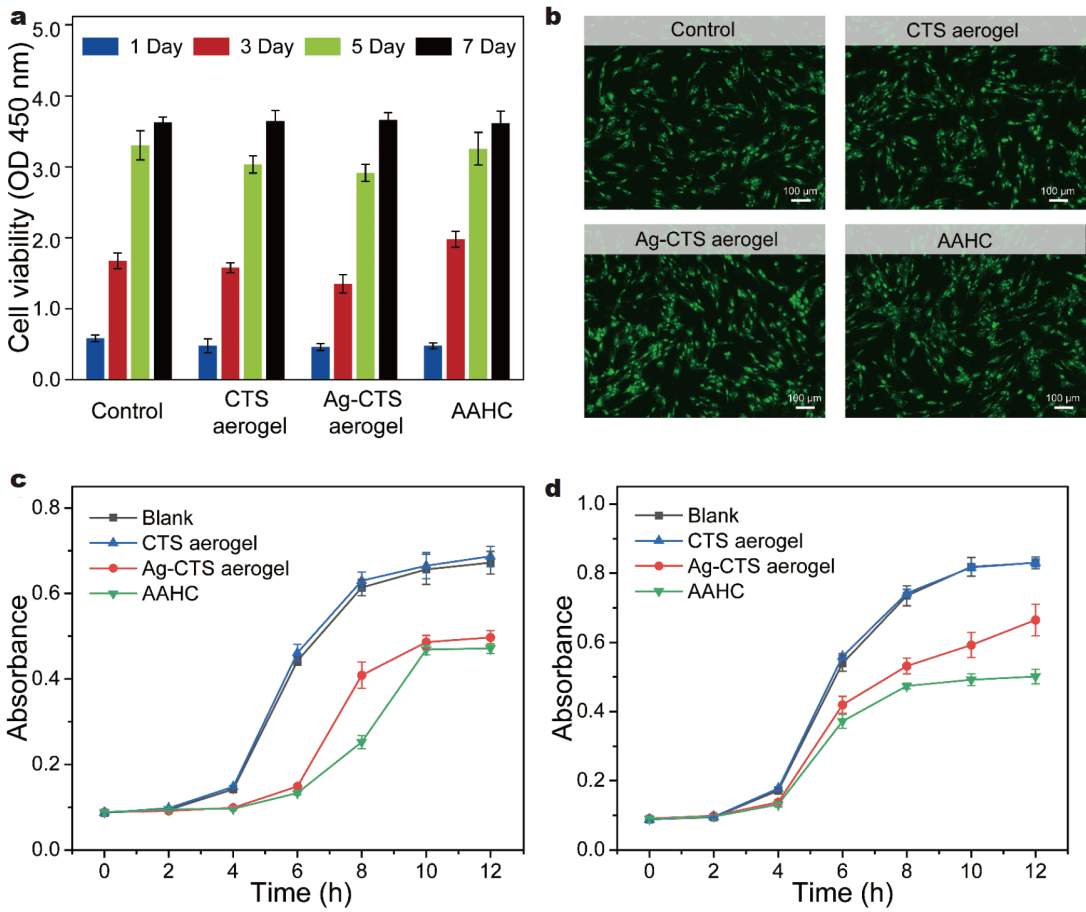

Figure 4 Cytocompatibility and anti-bacteria performance of AAHC. (a) Cell viability was compared among DMEM, CTS aerogel, Ag-CTS aerogel and AAHC conditioned media at 1, 3, 5, 7 days. (b) Fluorescent images of fibroblasts after 24-h culture in various conditioned media. Viability of (c) Gram-negative bacteria, E. coli and (d) Gram-positive bacteria, S. aureus after being co-cultured with blank medium, CTS aerogel, Ag-CTS aerogel and AAHC at continuous time points. Error bars represent SD; $N=5$.

above bacterial solution was measured. According to Fig. $4 \mathrm{c}, \mathrm{d}$, the $\mathrm{OD}_{600}$ values for both E. coli and S. aureus increased with incubation time because the bacteria proliferated over time. After incubation with CTS-AgNW and the hybrid, the $\mathrm{OD}_{600}$ value decreased significantly with time. In contrast, the $\mathrm{OD}_{600}$ values of E. coli and $S$. aureus in the CTS framework groups increased with the prolongation of culture time. These results demonstrated that AgNW integrated electronics can significantly inhibit the growth of E. coli and S. aureus, and their antibacterial properties were mainly derived from AgNWs. Unlike traditional adhesive conductors, this hybrid exhibits satisfactory antibacterial properties. More importantly, they can prevent infection when they are in direct contact with the skin, tissue surface and other organs of the human body.

Furthermore, in order to evaluate the in vivo histocompatibility of the hybrid, we implanted the CTS framework, CTS-AgNW aerogel and hybrid in a dorsal subcutaneous model of the rat (Fig. 5a). Histological assessment demonstrates that, after two weeks of implantation, three groups of subjects all generate a mild to moderate chronic inflammatory response involving macrophages, lymphocytes and occasional giant cells in association with the formation of a capsule of granulation tissue comprising fibroblasts, collagen and new blood vessels. There is no evidence of necrosis of the overlying skeletal muscle or skin, or of an eosinophilic response suggestive of an allergic reaction (Fig. 5b-g). Although the hybrid shows a higher degree of inflammatory response than the CTS framework and CTS-AgNW, as indicated by a denser chronic inflammatory reaction, no major damage to the surrounding dermal and muscular layers is observed after two weeks of implantation. The more pronounced inflammatory response of the hybrid might result from the faster bio-integration of the hydrogel coating and subsequent effects on the surrounding tissues.

\section{CONCLUSIONS}

In summary, we have demonstrated a new conductive aero-hydrogel hybrid with integration of conductivity and good mechanical properties. With the merits of wellorganized conductive AgNW architectures on the preprepared aerogel and aerogel-hydrogel interaction, this AAHC showed high electro-mechanical performance. In 

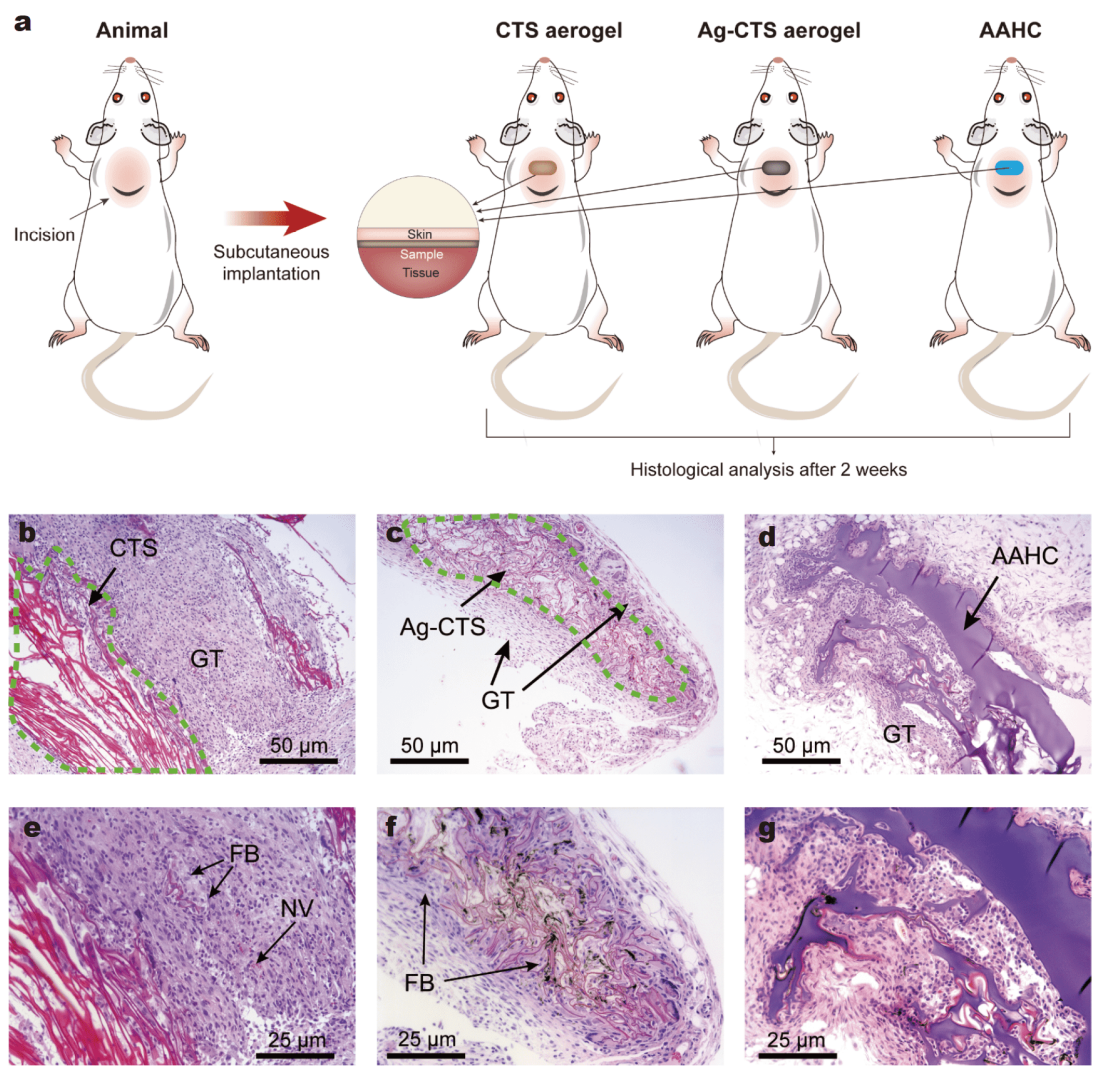

Figure 5 In vivo biocompatibility performance. (a) Schematic illustrations for in vivo biocompatibility implants in the rat dorsal subcutaneous pocket. Representative histological images stained with hematoxylin-eosin (H\&E) for assessment of the biocompatibility of subcutaneously implanted CTS aerogel (b, e), Ag-CTS aerogel (c, f) and AAHC (d, g) after two weeks. GT and FB indicate granulation tissue and fibrous capsule, respectively. All experiments were repeated three or four times with similar results.

addition, the hydrogel conductor with biocompatible and adhesive properties can firmly adhere to dynamic and wet biological surfaces for body signal detection, which is an indispensable performance to build electronic-biology interface. Furthermore, the inherent antibacterial property of the AAHC holds the promise for versatile applications, including soft implantable bio-electrodes and devices.

\section{Received 2 March 2021; accepted 23 March 2021;} published online 16 June 2021

1 Bai J, Wang R, Ju M, et al. Facile preparation and high performance of wearable strain sensors based on ionically cross-linked composite hydrogels. Sci China Mater, 2021, 64: 942-952

2 Guo T, Zhou D, Liu W, et al. Recent advances in all-in-one flexible supercapacitors. Sci China Mater, 2021, 64: 27-45

3 Deng J, Yuk $\mathrm{H}$, Wu J, et al. Electrical bioadhesive interface for bioelectronics. Nat Mater, 2020, 20: 229-236

4 Lee $\mathrm{GH}$, Moon $\mathrm{H}$, Kim $\mathrm{H}$, et al. Multifunctional materials for implantable and wearable photonic healthcare devices. Nat Rev Mater, 2020, 5: 149-165

5 Zhao J, Nyein HYY, Hou L, et al. A wearable nutrition tracker. Adv
Mater, 2021, 33: 2006444

6 Zhao C, Zhang P, Shi R, et al. Super-tough and strong nanocomposite fibers by flow-induced alignment of carbon nanotubes on grooved hydrogel surfaces. Sci China Mater, 2019, 62: 13321340

7 Zhu Z, Park HS, McAlpine MC. 3D printed deformable sensors. Sci Adv, 2020, 6: eaba5575

8 Liu X, Liu J, Lin S, et al. Hydrogel machines. Mater Today, 2020, 36: $102-124$

9 Yin X, Zhang Y, Xiao J, et al. Monolithic dual-material 3D printing of ionic skins with long-term performance stability. Adv Funct Mater, 2019, 29: 1904716

10 Sun JY, Keplinger C, Whitesides GM, et al. Ionic skin. Adv Mater, 2014, 26: 7608-7614

11 Liang S, Zhang Y, Wang H, et al. Paintable and rapidly bondable conductive hydrogels as therapeutic cardiac patches. Adv Mater, 2018, 30: 1704235

12 Tang $\mathrm{P}$, Yan $\mathrm{H}$, Chen L, et al. Anisotropic nanocomposite hydrogels with enhanced actuating performance through aligned polymer networks. Sci China Mater, 2020, 63: 832-841

13 Liao H, Guo X, Wan P, et al. Conductive MXene nanocomposite organohydrogel for flexible, healable, low-temperature tolerant strain sensors. Adv Funct Mater, 2019, 29: 1904507

14 Song P, Qin H, Gao HL, et al. Self-healing and superstretchable 
conductors from hierarchical nanowire assemblies. Nat Commun, 2018, 9: 2786

15 Gao HL, Xu L, Long F, et al. Macroscopic free-standing hierarchical 3D architectures assembled from silver nanowires by ice templating. Angew Chem Int Ed, 2014, 53: 4561-4566

16 Ahn $\mathrm{Y}$, Lee $\mathrm{H}$, Lee $\mathrm{D}$, et al. Highly conductive and flexible silver nanowire-based microelectrodes on biocompatible hydrogel. ACS Appl Mater Interfaces, 2014, 6: 18401-18407

17 Lee $\mathrm{P}$, Lee J, Lee $\mathrm{H}$, et al. Highly stretchable and highly conductive metal electrode by very long metal nanowire percolation network. Adv Mater, 2012, 24: 3326-3332

18 Zhang $\mathrm{Y}, \mathrm{He} \mathrm{P}$, Luo $\mathrm{M}$, et al. Highly stretchable polymer/silver nanowires composite sensor for human health monitoring. Nano Res, 2020, 13: 919-926

19 Kim KK, Hong S, Cho HM, et al. Highly sensitive and stretchable multidimensional strain sensor with prestrained anisotropic metal nanowire percolation networks. Nano Lett, 2015, 15: 5240-5247

20 Jeong CK, Lee J, Han S, et al. A hyper-stretchable elastic-composite energy harvester. Adv Mater, 2015, 27: 2866-2875

21 Jung J, Cho H, Yuksel R, et al. Stretchable/flexible silver nanowire electrodes for energy device applications. Nanoscale, 2019, 11: 20356-20378

22 Zare Y, Shabani I. Polymer/metal nanocomposites for biomedical applications. Mater Sci Eng-C, 2016, 60: 195-203

23 Altinkok C, Oytun F, Basarir F, et al. Cysteamine-functionalized silver nanowires as hydrogen donor for type II photopolymerization. J Photochem Photobiol A-Chem, 2017, 346: 479-484

24 Schiavone G, Lacour SP. Conformable bioelectronic interfaces: Mapping the road ahead. Sci Transl Med, 2019, 11: eaaw5858

25 Chang EI, Galvez MG, Glotzbach JP, et al. Vascular anastomosis using controlled phase transitions in poloxamer gels. Nat Med, 2011, 17: 1147-1152

26 Li J, Celiz AD, Yang J, et al. Tough adhesives for diverse wet surfaces. Science, 2017, 357: 378-381

27 Gao HL, Lu Y, Mao LB, et al. A shape-memory scaffold for macroscale assembly of functional nanoscale building blocks. Mater Horiz, 2014, 1: 69-73

28 Wang JL, Lu YR, Li HH, et al. Large area co-assembly of nanowires for flexible transparent smart windows. J Am Chem Soc, 2017, 139: 9921-9926

29 Yang J, Bai R, Suo Z. Topological adhesion of wet materials. Adv Mater, 2018, 30: 1800671

30 Annabi N, Zhang YN, Assmann A, et al. Engineering a highly elastic human protein-based sealant for surgical applications. Sci Transl Med, 2017, 9: eaai7466

31 Yuk H, Varela CE, Nabzdyk CS, et al. Dry double-sided tape for adhesion of wet tissues and devices. Nature, 2019, 575: 169-174

Acknowledgements This work was supported by the National Natural Science Foundation of China (51732011, 51702310, 21431006, and 21761132008), the Foundation for Innovative Research Groups of the National Natural Science Foundation of China (21521001), the Key Research Program of Frontier Sciences, CAS (QYZDJ-SSW-SLH036), the Users with Excellence and Scientific Research Grant of Hefei Science Center of CAS (2015HSC-UE007), and Anhui Provincial Natural Science Foundation (1808085ME115).

Author contributions $\quad \mathrm{Yu} \mathrm{SH}$ and Pan $\mathrm{Z}$ conceived the idea and designed the experiments. Yu SH supervised the project and wrote the paper. Pan $\mathrm{Z}$ and Wang ZY designed and carried out the experiments, performed the experiments, collected and analyzed the data, and wrote the paper. Wang $\mathrm{MH}$ and Yang L designed and carried out the in vitro and in vivo antibacterial and biocompatibility experiments. All authors discussed the results.

Conflict of interest The authors declare that they have no conflict of interest.

Supplementary information Supporting data are available in the online version of the paper.

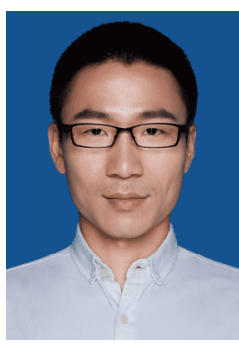

Zhao Pan received his bachelor degree in pharmacy from Lanzhou University, China in 2012. Since then he has been conducting research at the University of Science and Technology of China (USTC) under the supervision of Prof. Shu-Hong Yu. His research interest is the design and fabrication of biomedical materials.

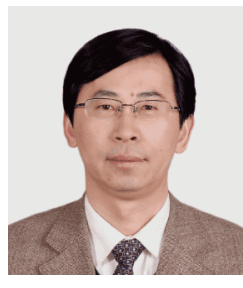

Shu-Hong Yu completed his $\mathrm{PhD}$ from the USTC. He was a postdoctoral fellow with Prof. Masahiro Yoshimura (Tokyo Institute of Technology) and a Humboldt fellow with Prof. Markus Antonietti and Helmut Cölfen (MPI of Colloids and Interfaces, Germany). In 2002, he was appointed the Cheung Kong Professor at USTC. Currently, he leads the Division of Nanomaterials \& Chemistry at Hefei National Laboratory for Physical Sciences at the Microscale, USTC. He was elected as an academician of Chinese Academy of Sciences in 2019. His current research interests include bio-inspired synthesis and self-assembly of new nanostructured materials and nanocomposites, and their related properties.

\section{由银纳米线组装结构制备自粘性气-水凝胶复合 导体}

潘㻇 $^{1}$, 王泽宇 ${ }^{1}$, 王默涵 ${ }^{2}$, 杨路 ${ }^{2}$, 俞书宏 ${ }^{*}$

摘要 具有粘合性能的导电水凝胶是一种非常有前景的生物电子 材料, 其中金属纳米材料通常被用作添加材料以提高水凝胶的导 电性. 然而, 由于纳米导电材料在水凝胶中极容易聚集, 使得高导 电率的金属纳米材料复合水凝胶难以制备. 本文中, 我们通过在壳 聚糖-银纳米线复合气凝胶中原位形成水凝胶网络, 设计并制备了 一种可导电、可拉伸、可粘合、可抗菌的新型多功能水凝胶. 高 导电性能的银纳米线在具有形状记忆性能的气凝胶内表面集成为 导电通路, 并且气凝胶还对复合水凝胶起到增强、增韧的效果, 银 离子固有杀菌能力也使得该导电水凝胶具有优异的抗菌性能. 最 后, 灌注在气凝胶中的水凝胶分子链上的活性官能团, 使得复合水 凝胶可以通过分子缝线牢固地结合在湿态生物组织表面. 本文提 出的这种气凝胶辅助导电水凝胶组装的新策略, 为实现将功能单 元与水凝胶组装及复合提供了一条新途径 